\title{
Krüppel-Like Factor 2 Regulates Degradation of Type II Collagen by Suppressing the Expression of Matrix Metalloproteinase (MMP)-13
}

\author{
Yanhao Yuan Honglue Tan Pengyi Dai \\ The Third Department of Knee's Injury, Henan Luoyang Orthopedic-Traumatological Hospital( Henan \\ Province Orthopedic Hospital), Luoyang, Henan, China
}

\section{Key Words}

Krüppel-like factor 2 (KLF2) - Matrix metalloproteinases (MMP)-13 - Type II collagen • Interleukin-1 $1 \beta$ Osteoarthritis

\begin{abstract}
Background/Aims: Krüppel-like factor 2 (KLF2) plays an essential role in the inhibition of endothelial cell and macrophage activation during the inflammatory process. However, the roles of KLF2 in chondrocytes and the pathological progression of osteoarthritis $(O A)$ remain unknown. The aim of this study was to investigate the function of KLF2 in the inhibition of cartilage matrix destruction in chondrocytes. Methods: RT-PCR and western blot analysis was used to determine the expression of KLF2 in human chondrocytes. Luciferase assay, ELISA assay and MMP-13 enzymatic activity assays were used to investigate the effects of KLF2 in regulating MMP-13 expression. Western blot analysis was used to examine the effects of KLF2 in suppressing degradation of type II collagen. Results: KLF2 is expressed in primary chondrocytes and is downregulated in OA chondrocytes. Expression of KLF2 in primary chondrocytes was reduced in response to IL-1 $\beta$. Overexpression of KLF2 robustly inhibited IL-1 $\beta$-induced MMP-13 expression. Conversely, knockdown of KLF2 markedly exacerbated MMP-13 expression. Mechanistically, KLF2 could suppress the activation of MMP-13 promoter. However, knockdown of KLF2 could promote the activation of MMP-13 promoter. Importantly, overexpression of KLF2 ameliorated the degradation of type II collagen while silencing of KLF2 exacerbated the degradation of type II collagen induced by IL-1ß. Conclusions: KLF2 may be a potential therapeutic target for OA treatment.

\section{Introduction}

Degradation of articular cartilage is an important hallmark of osteoarthritis (OA) [1]. Type II collagen and aggrecan in chondrocytes are the two main components of the 


\section{Cellular Physiology Cell Physiol Biochem 2017;42:2159-2168

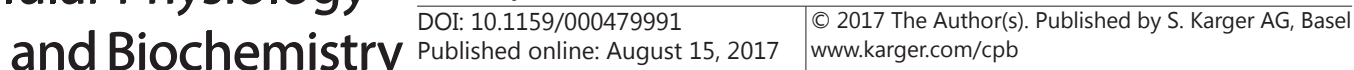 \\ Yuan/Tan/Dai: KLF2 Regulates Degradation of Type II Collagen}

cartilage extracellular matrix, which play essential roles in maintaining cartilage integrity [2]. Matrix metalloproteinases (MMPs) are a family of enzymes that mediate irreversible matrix degradation and subsequent joint destruction in OA. Irreversible degradation of type II collagen mediated by MMP-13 occurs early in the pathogenesis of OA. MMP-13 expression co-localizes with type II collagen degradation in active OA lesions. Moreover, OA chondrocytes express MMP-13, which efficiently cleaves type II collagen [3]. Pro-inflammatory cytokines such as interleukin-1 $\beta$ (IL-1 $\beta$ ) have been shown to be involved in the progression of OA [4]. The role of IL-1 $\beta$ in cartilage degeneration is associated with its ability to induce apoptosis in human articular chondrocytes (HACs) [5]. IL-1 $\beta$ up-regulates MMP-13 expression and triggers the inflammatory process [6]. Notably, IL-1 $\beta$ promotes degradation of certain components of the cartilage extracellular matrix, such as type II collagen, and disrupts cartilage integrity [7]. Therefore, disrupting the activity of pro-inflammatory cytokines and subsequent MMP-13 expression has become an important strategy for reducing cartilage degradation in $\mathrm{OA}[8,9]$.

Krüppel-like factors (KLFs) are a subclass of the zinc finger family of DNA-binding transcription factors that share homology with the Drosophila gene Krüppel [10]. Many KLFs contain zinc finger domains located at the C-terminus, which bind to similar DNA sequences, such as the "CACCC" elements or the "GT box." [11]. Krüppel-like factor 2 (KLF2) is a member of the KLF family [12]. KLF2 is expressed in T cells and vascular endothelial cells (ECs) [13]. KLF2 has an anti-atherosclerotic and anti-inflammatory capacity in ECs in response to proinflammatory cytokines. The pro-inflammatory cytokine IL- $1 \beta$ has been reported to inhibit the expression of KLF2, while KLF2 suppresses the induction of vascular cell adhesion molecule-1 (VCAM-1) and the endothelial adhesion molecule E-selectin in response to various proinflammatory stimuli [14]. However, it remains unknown whether KLF2 is expressed in chondrocytes. In addition, the role of KLFs in OA inflammation remains to be defined.

\section{Materials and Methods}

\section{Cell culture and treatment}

HUVECs were purchased from Lonza (Walkersville, USA). Cells were cultured in EBM-2 media with supplemental growth factors in accordance with manufacturer's instructions. The mouse chondrogenic cell line ATDC5 was obtained from RIKEN. Human chondrogenic cell line OUMS27 was purchased from the Health Science Research Resources Bank. ATDC5 and OUMS27 cells were cultured in Dulbecco's modified Eagle's medium (DMEM) containing $10 \%$ fetal bovine serum (FBS). Cells were maintained at $37^{\circ} \mathrm{C}$ in a humidified atmosphere with $5 \% \mathrm{CO}_{2}$. OA cartilage samples were collected from eight OA patients. Normal samples were collected from ten patients with femoral neck fractures who underwent femoral head replacement surgeries. Samples were washed 3 times with PBS and then cut into small pieces, followed by sequential digestion of the extracellular matrix with pronase $(0.15 \%, \mathrm{w} / \mathrm{v})$ for $2 \mathrm{~h}$ and collagenase $(0.2 \%, \mathrm{w} / \mathrm{v})$ overnight at $37^{\circ} \mathrm{C}$. After centrifugation at $1000 \times \mathrm{g}$ for $15 \mathrm{~min}$, cells were suspended in DMEM/Ham's 12 supplemented with $10 \%(\mathrm{v} / \mathrm{v})$ fetal calf serum (FCS), $2 \mathrm{mM} \mathrm{L-glutamine,} \mathrm{penicillin}(0.1 \mathrm{U} / \mathrm{ml})$, streptomycin $(100 \mathrm{ng} / \mathrm{ml})$, and $250 \mathrm{ng} / \mathrm{ml}$ amphotericin B. Isolated chondrocytes were seeded in $75 \mathrm{~cm}^{2}$ culture flasks at a density of 2.5 $\times 10^{4}$ cells $/ \mathrm{cm}^{2}$. The study was approved by the ethics committee of Luoyang Orthopedic Hospital, Henan. Samples were collected after participants submitted the required written informed consent.

Real-time polymerase chain reaction (PCR)

Total RNA from cells were isolated using Qiazol reagent (Qiagen, USA). RNA was reverse-transcribed into cDNA using a Revert Aid First Strand cDNA Synthesis kit (ABI, USA). cDNA was used for real-time PCR on an ABI Prism 7000 sequence detection system (ABI, USA) using SYBR Green PCR Master Mix (Qiagen) in accordance with the manufacturer's instructions [15]. The relative abundance of mRNA from the perspective target genes was calculated after normalization to GAPDH. The following primers were used in this study:

Human KLF2: forward, 5'-TGCGGCAAGACCTACACCAAGAGT-3'; reverse, 5'-AGCCGCAGCCGTCCCAGTT-3'; human MMP-13: forward, 5'-CCAGTCTCCGAGGAGAAACA-3'; reverse, 5'-AAAAACAGCTCCGCATCAAC-3'; 


\section{Cellular Physiology Cell Physiol Biochem 2017;42:2159-2168 \begin{tabular}{c|c|c|} 
DOI: 10.1159/000479991 & C 2017 The Author(s). Published by S. Karger AG, Basel \\
www.karger.com/cpb
\end{tabular} \\ Yuan/Tan/Dai: KLF2 Regulates Degradation of Type II Collagen}

human GAPDH: forward, 5'-CCACATCGCTCAGACACCAT-3'; reverse, 5'-CCAGGCGCCCAATACG-3'. cDNA content of the target genes in each group was normalized to GAPDH. The relative expression levels of the target genes were analyzed using the comparative threshold cycle $\left(2^{-\Delta \Delta \mathrm{ct}}\right)$ method.

\section{Western blot analysis}

Cells were lysed using RIPA buffer with a protease and phosphatase inhibitor cocktail (Sigma-Aldrich). Equal amounts of proteins $(15 \mu \mathrm{g})$ were subjected to $10 \%$ SDS-polyacrylamide gel electrophoresis and then electrotransferred onto polyvinylidene difluoride (PVDF) membranes. Membranes were probed with primary antibodies overnight at $4^{\circ} \mathrm{C}$ followed by incubation with horseradish peroxidase (HRP)-conjugated secondary antibodies (1:5000) for $60 \mathrm{~min}$. Blots were developed using a western chemiluminescence system. The following antibodies were used in this study: rabbit polyclonal anti-KLF2 antibody (1: 1000, \#ab203591, Abcam, USA); rabbit polyclonal anti-GAPDH antibody (1:3000, \#G9545, Sigma-Aldrich); mouse monoclonal anti- $\beta$-actin antibody (1: 10000, sc-130300, Santa Cruz Biotechnology, USA); rabbit polyclonal anti-collagen II antibody (1: 1000, \#ab34712, Abcam, USA).

\section{Immunocytochemistry}

Cells were cultured on chamber slides. After washing 3 times with PBS, cells were fixed in $4 \%$ formaldehyde for $30 \mathrm{~min}$ at room temperature (RT). After blocking with $2 \%$ bovine serum albumin (BSA) for $1 \mathrm{~h}$ at RT, cells were incubated with rabbit anti-human KLF2 (1:200, Abcam) overnight at $4^{\circ} \mathrm{C}$, followed by TRITC conjugated goat anti-rabbit IgG (1:100, Santa Cruz) for $2 \mathrm{~h}$. Nuclei were stained with DAPI (4',6'-diamidino-2-phenylindole dihy-drochloride), and staining was visualized using fluorescence microscopy (Olympus, Tokyo, Japan).

\section{Luciferase assay and reporter construct}

The luciferase reporter vector pGL3 basic (Promega) was used to construct a plasmid containing the proximal $370 \mathrm{bp}$ of the human MMP-13 promoter. The MMP-13 promoter construct $\mathrm{p} 370$-Luc was generated by PCR using a 5'-sense primer containing the appropriate MMP-13 promoter sequence using the following primers: 5' -CCAACAGTACCG-GAATGCCAAGC-3' (forward) and 5'-CGGGTACCATGGGCGTACACATA-3' (reverse). Human KLF2 and a non-specific control siRNA were acquired from Invitrogen, USA and packaged into lentiviruses in accordance with the manufacturer's instructions. Human lentiviral KLF2 was purchased from ABM, Inc., CA. Human chondrocytes were transiently transfected with MMP-13 reporter and $\beta$-galactosidase vector (\#E1081, Promega). Cells were seeded with about $80 \%$ confluence into a 24well plate at a density of $1 \times 10^{5}$ per well. Then, $2.5 \mu \mathrm{l}$ lipofectamine reagent (Lipofectamine 2000, USA) was diluted in $50 \mu \mathrm{l}$ Opti-MEM medium, and $1 \mu \mathrm{g}$ DNA ( $\beta$-galactosidase vector and MMP-13 reporter plasmid) was diluted with $50 \mu \mathrm{l}$ Opti-MEM medium. Equal volumes $(50 \mu \mathrm{l})$ of DNA-lipid complex and lipofectamine reagent were mixed at a ratio of 1:1. The mixture was incubated for $5 \mathrm{~min}$ at RT and then added to cells. Transfected cells were incubated for 48 hours at $37^{\circ} \mathrm{C}$. Luciferase activity was measured and normalized for transfection efficiency using $\beta$-galactosidase activity.

\section{Enzyme linked immunosorbent assay (ELISA) assay}

Culture medium was collected for ELISA assay using a MMP-13 ELISA commercial kit (\#DM1300, R\&D Systems) in accordance with the manufacturer's instructions. Then, $100 \mu \mathrm{l}$ assay buffer was added to the 96well solid phase sandwich ELISA plate, and $50 \mu \mathrm{l}$ sample or control standard was added and incubated for 2 $\mathrm{h}$ at RT on a shaker. After washing 3 times, $200 \mu \mathrm{L}$ of conjugate was added to each well and incubated for 2 $\mathrm{h}$ at RT. Next, $200 \mu \mathrm{l}$ substrate solution was added and incubated at RT for $30 \mathrm{~min}$. Then, $50 \mu \mathrm{l}$ stop solution was added into each well. Absorbance recorded at $450 \mathrm{~nm}$ was used to represent the concentration of MMP13. Results were indexed as a relative value by normalization to the untreated control group.

MMP-13 enzymatic activity assays

Cell culture supernatants were collected to determine MMP-13 activity using a fluorogenic substrate Mca-Pro-Leu-Gly-Leu-Dpa-Ala-Arg-NH2 (Calbiochem, UK) [16]. Briefly, $20 \mathrm{ml}$ of culture supernatant was mixed with an equal amount of 4- aminophenylmercuric acetate (1.5 mM) (APMA, Sigma-Aldrich) diluted in Tris- $\mathrm{HCl}$ reaction buffer. Fluorogenic MMP-13 substrate was then added to the reaction system at a final concentration of $10 \mathrm{mM}$ and incubated for $4 \mathrm{~h}$ in darkness at $37^{\circ} \mathrm{C}$. The resulting fluorescence signals were 


\section{Cellular Physiology Cell Physiol Biochem 2017;42:2159-2168 and Biochemistry \begin{tabular}{l|l} 
DOI: 10.1159/000479991 & $\begin{array}{l}\text { C } 2017 \text { The Author(s). Published by S. Karger AG, Basel } \\
\text { www.karger.com/cpb }\end{array}$
\end{tabular}

Fig. 1. Expression of KLF2 in cartilage cells. (A) mRNA expression of KLF2 and glyceraldehyde 3-phosphate dehydrogenase (GAPDH) serving as the housekeeping gene determined by RT-PCR in human primary chondrocytes and chondrogenic cell lines ATOCS and OUMS27 cells with HUVECs used as a positive control; (B) Western blot analysis revealed that KLF2 was expressed in human primary chondrocytes and chondrogenic cell lines ATOCS and OUMS27 cells. GAPDH was used as an endogenous control. Experiments were repeated in triplicate.

assessed using a Fluostar Optima spectrofluorimeter at an excitation wavelength of $325 \mathrm{~nm}$ and an emission wavelength of $393 \mathrm{~nm}$. Results were indexed using arbitrary units of fluorescence and were normalized to the uncontrolled group.

\section{Statistical analysis}

Data are presented as means \pm standard error of mean (SEM). Statistical analysis was performed by a two-tailed student's t-test for independent samples or analysis of variance (ANOVA) for multiple comparisons. $P$ values less than 0.05 were considered statistically significant.

\section{Results}

To study the involvement of KLF2 in cartilage metabolism, the RT-PCR method was used to determine the expression of KLF2 in cartilage cells at the mRNA level. The results in Fig. 1A show that KLF2 was expressed in primary chondrocytes. Additionally, it was found that KLF2 is expressed in cells from the ATDC5 and OUMS27 chondrogenic cell lines. HUVECs were used as a positive control. KLF2 expression in primary chondrocytes and chondrogenic ATDC5 and OUMS27 cells was confirmed by western blot analysis (Fig. 1B).

We next investigated whether KLF2 is involved in cartilage metabolism dysfunction in OA patients. KLF2 expression in normal and $\mathrm{OA}$ chondrocytes was determined by realtime PCR. As shown in Fig. 2A, KLF2 mRNA was significantly lower in $\mathrm{OA}$ chondrocytes as compared to normal chondrocytes. Immunofluorescence was used to stain chondrocytes with KLF2 antibody. The results in Fig. 2B display that KLF2 was localized in the nuclei of chondrocytes. Importantly,
(A)

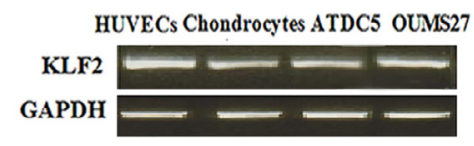

(B)

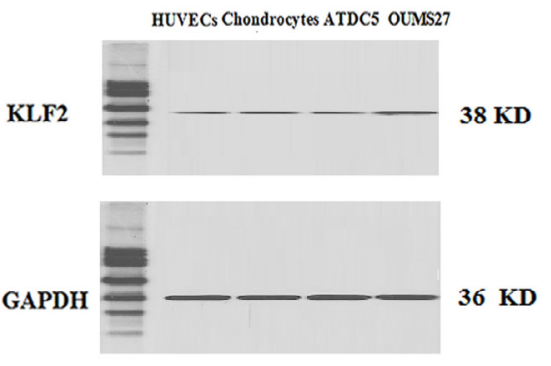

(A)

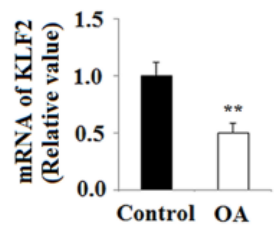

(B)

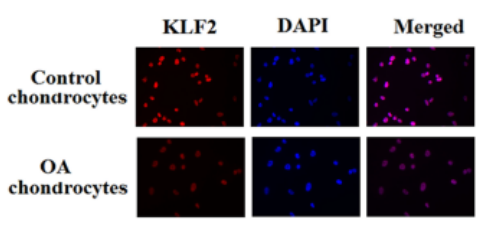

(C)

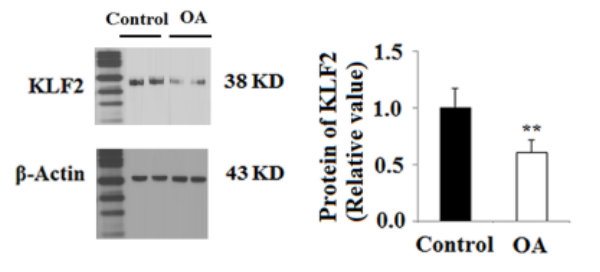

Fig. 2. Expression of KLF2 is reduced in human $\mathrm{OA}$ chondrocytes. Chondrocytes were isolated from 10 normal and 8 OA cartilage samples. (A) mRNA expression of KLF2 and glyceraldehyde 3-phosphate dehydrogenase (GAPDH) serving as the housekeeping gene determined by real time-PCR $(* *$, $\mathrm{P}<0.01$ vs. control group); (B) Expression of KLF-2 at the protein level in normal and OA chondrocytes was assayed by immunofluorescence; (C) Representative bands of KLF- 2 expression in normal and OA chondrocytes determined by western blot analysis (student t-test, ${ }^{* *}, \mathrm{P}<0.01$ vs. control group). 

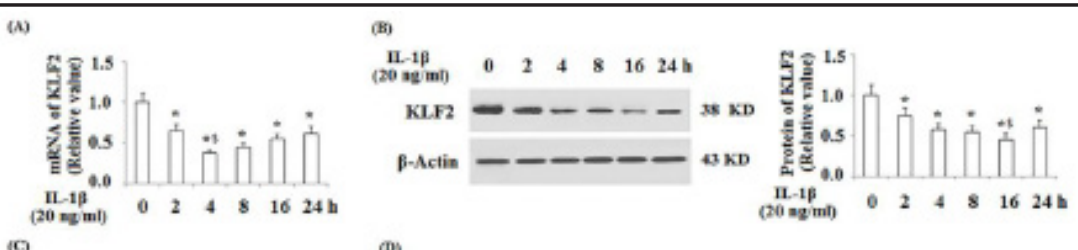

(C)

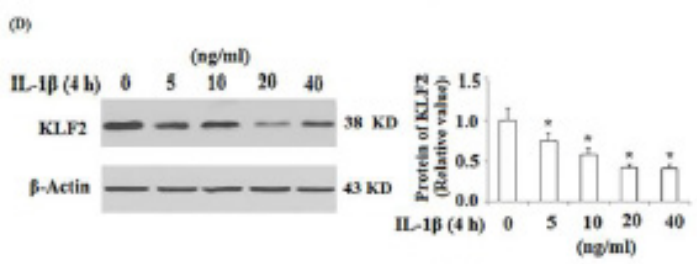

(5)

क)
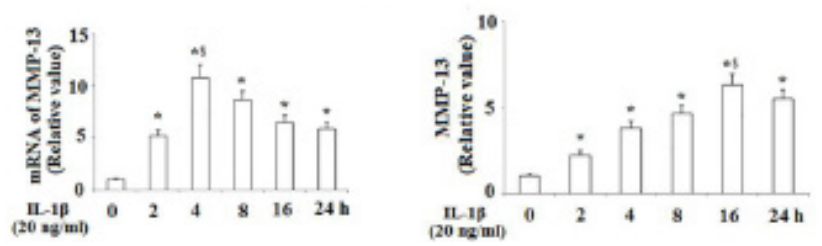

(G)

Fig. 3. Expression of KLF2 and MMP-13 in human primary chondrocytes in response to IL-1 $\beta$. KLF2 is reduced by IL-1 $\beta$ in a time- and dose-dependent manner. $\beta$-actin was used as an endogenous control for western blot analysis. (A). Primary chondrocytes were incubated with $20 \mathrm{ng} / \mathrm{mL}$ IL-1 $\beta$ for the indicated time periods. Expression of KLF2 at the mRNA level was assessed by real-time PCR analysis. Minimal levels of KLF2 at the mRNA level were observed after $4 \mathrm{~h}$ incubation with IL-1 $\beta\left({ }^{*}, \mathrm{P}<0.05\right.$ vs. untreated control group, $\$, P<0.05$ vs. other time points, $n=5-6$ ); (B) Expression of KLF2 at the protein level was assessed by western blot analysis. Minimal levels of KLF2 at the protein level was observed after $4 \mathrm{~h}$ and $16 \mathrm{~h}$ incubation with IL-1 $\beta$; (C) Human primary chondrocytes were incubated with varying doses of IL-1 $\beta$, and expression of KLF2 at the mRNA level was assessed by real-time PCR after $4 \mathrm{~h}$; (D) Human primary chondrocytes were incubated with varying doses of IL-1 $1 \beta$, and expression of KLF2 at the protein level was assessed by western blot analysis after $16 \mathrm{~h}$. (E). The time course of MMP-13 expression at the mRNA level in human primary chondrocytes in response to $20 \mathrm{ng} / \mathrm{mL} \mathrm{IL-1} \beta$ was assessed by real-time PCR. Results were normalized to the untreated group. (F) The time course of MMP-13 expression at the protein level in human primary chondrocytes in response to $20 \mathrm{ng} / \mathrm{mL}$ IL-1 $\beta$ was assessed by ELISA analysis. Results were normalized to the untreated group; (G) The time course of MMP-13 activity. Results were normalized to the untreated group (ANOVA, *, $\mathrm{P}<0.05$ vs. untreated control group, $\$, \mathrm{P}<0.05$ vs. other time points, $\mathrm{n}=5-6$ ).

illumination of KLF2 staining was weaker in chondrocytes from OA cartilage than in those from normal cartilage. Western blot analysis revealed that the level of KLF2 was significantly lower in chondrocytes from OA cartilage than in those from normal cartilage (Fig. 2C).

Pro-inflammatory cytokines are critical contributors in the pathogenesis of OA. Interestingly, KLF2 expression has been reported to be reduced by pro-inflammatory stimuli, such as IL-1 $\beta$, in endothelial cells [14]. However, it remains unknown whether pro-inflammatory cytokines influence KLF2 expression in chondrocytes. To investigate the influence of pro-inflammatory cytokines on the expression of KLF2, chondrocytes were treated with IL-1 $\beta$ for various periods of time and with various doses. mRNA and protein expression of KLF2 was examined using real-time PCR and western blot analysis, respectively. The results indicate that expression of KLF2 at both the mRNA (Fig. 3A) and protein levels (Fig. 3B) was significantly reduced in chondrocytes incubated with IL-1 $\beta$ (20 $\mathrm{ng} / \mathrm{mL}$ ) within $24 \mathrm{~h}$. Minimal mRNA and protein levels of KLF2 were observed after $4 \mathrm{~h}$ and $16 \mathrm{~h}$ incubation with IL-1 $\beta$. In addition, IL-1 $\beta$ reduced the expression of KLF2 at both the mRNA (Fig. 3C) and protein levels in a dose-dependent manner (Fig. 3D). MMP-13, a matrix metalloproteinase, is an especially important catabolic factor for ECM degradation in OA. We analyzed the influence of IL-1 $\beta$ on MMP-13 expression in chondrocytes. The results in Fig. 3E 
(A)

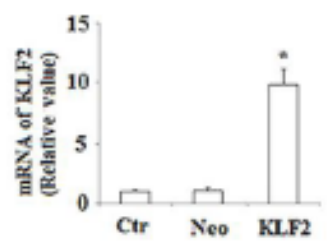

(C)

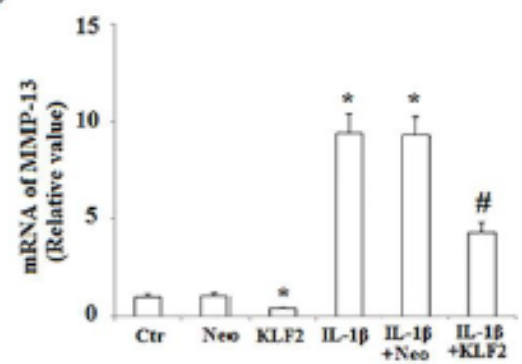

(B)

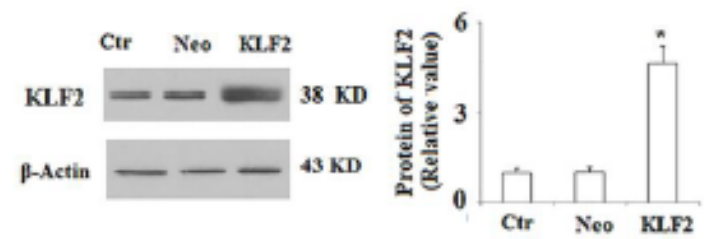

(D)

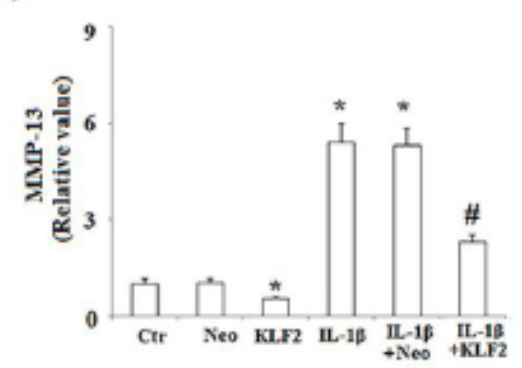

Fig. 4. KLF2 overexpression reduces the expression of MMP-13 in human primary chondrocytes. Human primary chondrocytes were transiently infected with lentiviral KLF2 (KLF2) or empty vector (Neo) for 24 $\mathrm{h}$ and then incubated with IL-1 $\beta(20 \mathrm{ng} / \mathrm{mL})$. (A) KLF2 expression was assessed at the mRNA level by real-time PCR; (B) KLF2 expression was assessed at the protein level by western blot analysis. $\beta$-actin was used as an endogenous control; (C) MMP-13 expression at the mRNA level was assessed after treatment with IL-1 $\beta$ for $4 \mathrm{~h}$ by real-time PCR; (D) MMP-13 expression at the protein level was assessed after treatment with IL-1 $\beta$ for $16 \mathrm{~h}$ by ELISA analysis. Results were normalized to the untreated group (ANOVA, *, $P<0.05$ vs. untreated cells (Ctrl); \#, $P<0.05$ vs. IL- $1 \beta$ group, $\mathrm{n}=6$ ).

demonstrate that levels of MMP-13 mRNA in chondrocytes were obviously augmented after incubation with IL-1 $\beta$ for 2 to $24 \mathrm{~h}$ and peaked at $4 \mathrm{~h}$. Similarly, levels of MMP-13 protein also increased significantly after incubation with IL-1 $\beta$ for 4 to $24 \mathrm{~h}$, and peaked at $16 \mathrm{~h}$ (Fig. 3F). In addition, immunoassay analysis of culture supernatant demonstrated that stimulation of primary human chondrocytes with IL- $1 \beta$ increased MMP-13 activity in a dose-dependent manner, which peaked at $16 \mathrm{~h}$, and is positively correlated with the amount of protein but not with the amount of mRNA (Fig. 3G).

There was an obvious negative correlation between the increase in MMP-13 and the reduction in KLF2 induced by IL-1 $\beta$ in chondrocytes, suggesting a relationship between these two proteins. Here, we examined the effect of KLF2 on IL-1 $\beta$-induced elevation of MMP- 13. Chondrocytes were infected with lentiviral KLF2 for $24 \mathrm{~h}$, followed by treatment with IL$1 \beta$. Successful overexpression of KLF2 at the mRNA and protein levels is shown in Fig. 4A and Fig. 4B, respectively. Real-time PCR and ELISA results indicate that KLF2 significantly ameliorated the expression of MMP-13 at the mRNA and protein levels (Fig. 4C and 4D) in the presence or absence of IL-1 $\beta$. To further evaluate the role of KLF2 in regulating MMP-13 expression, the expression of KLF2 in chondrocytes was knocked down by siRNA. Successful knockdown of KLF2 is shown in Fig. 5A and 5B. The results in Fig. 5C and 5D demonstrate that the elevation of MMP-13 induced by IL-1 $\beta$ was potently enhanced by silencing of KLF2. Taken together, these data suggest that KLF2 can regulate MMP-13 expression in chondrocytes.

To understand the mechanism by which KLF2 regulates MMP-13 gene expression in chondrocytes, we investigated the effects of inactivation of the MMP-13 promoter using a luciferase assay. As shown in Fig. 6A, IL-1 $\beta$ significantly increased the promoter activity of MMP-13, which was ameliorated by overexpression of KLF2. In contrast, MMP-13 promoter activity was significantly higher in cells transfected with KLF2 siRNA both in the presence and absence of IL-1 $\beta$ stimulation (Fig. 6B) $(P<0.05)$.

Degradation of components of the ECM, such as type II collagen, is one of the most important pathological characteristics of OA. MMP-13 is a major executor of type II collagen 
(A)

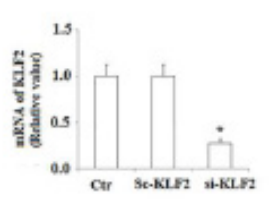

(C)

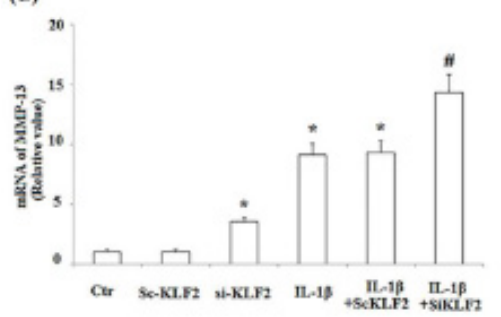

(B)

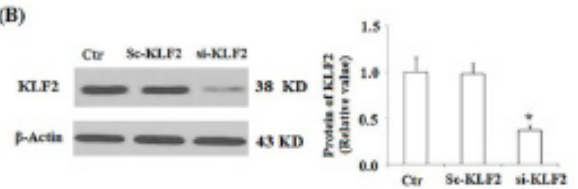

(D)

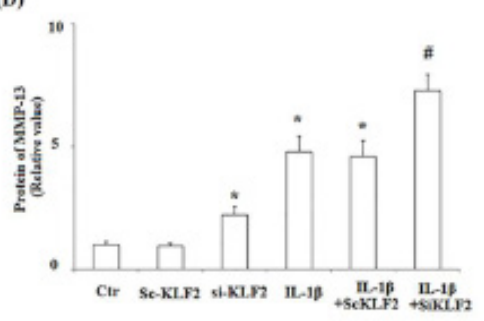

Fig. 5. Effect of KLF2 knockdown on MMP-13 expression in human primary chondrocytes. Human primary chondrocytes were transiently transfected with lentiviral KLF4 siRNA oligonucleotide (si-KLF4) or KLF4 scrambled siRNA (sc-KLF2) for $24 \mathrm{~h}$ and then incubated with IL-1 $\beta(20 \mathrm{ng} / \mathrm{mL}$ ). (A) KLF2 inhibition was assessed at the mRNA level by real-time PCR; (B) KLF2 inhibition was assessed at the protein level by western blot analysis. $\beta$-actin was used as an endogenous control for western blot analysis. (C) MMP-13 expression at the mRNA level was assessed after treatment with IL-1 $\beta$ for $4 \mathrm{~h}$ by real-time PCR; (D) MMP-13 expression at the protein level was assessed after treatment with IL-1 $\beta$ for $16 \mathrm{~h}$ by ELISA analysis. Results were normalized to the untreated group (ANOVA, ${ }^{*}, P<0.05$ vs. untreated cells (Ctrl); $\#, P<0.05$ vs. IL-1 $\beta$ group, n=6).

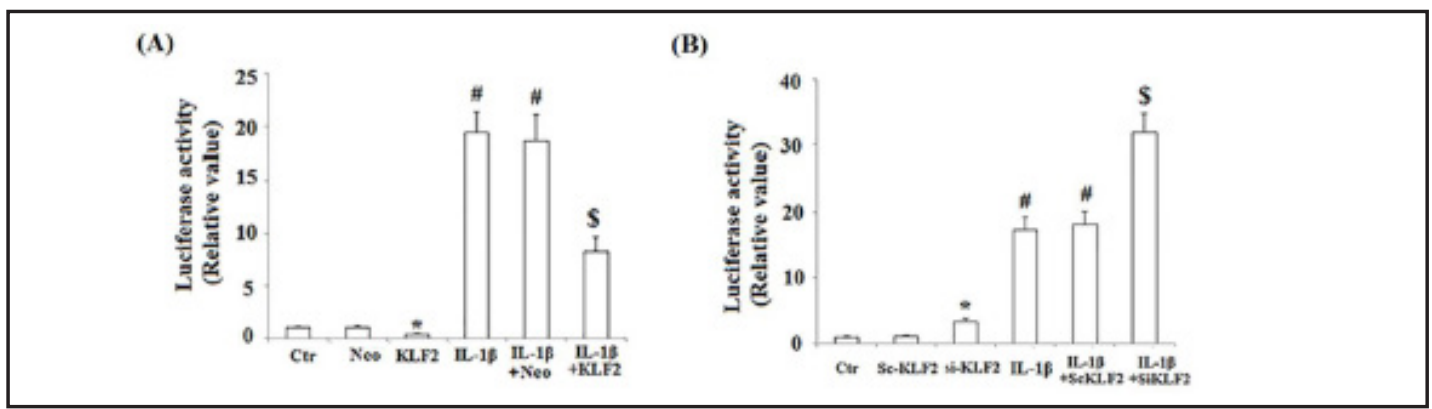

Fig. 6. KLF2 regulates MMP-13 promoter activity in chondrocytes. (A) Chondrocytes were co-transfected in triplicate with the indicated MMP-13 promoter luciferase plasmids, lentiviral KLF2, or control vector (Neo) for $48 \mathrm{~h}$ and then incubated with IL-1 $\beta$ (20 ng/mL) for $12 \mathrm{~h}$. Transcriptional activity was detected by Dual Luciferase assay; (B) Chondrocytes were co-transfected in triplicate with the indicated MMP-13 promoter luciferase plasmids, lentiviral siKLF2, or control scramble RNA interference (Sc-KLF2) for $48 \mathrm{~h}$ and then incubated with IL-1 $\beta(20 \mathrm{ng} / \mathrm{mL})$ for $12 \mathrm{~h}$. Transcriptional activity was detected by Dual Luciferase assay (ANOVA, * , \#, $P<0.05$ vs. untreated cells (Ctrl); $\$, P<0.05$ vs. IL-1 $\beta$ group, $n=5-6$ ).

degradation. Thus, we investigated the effects of KLF2 on type II collagen degradation. Our results indicate that IL- $1 \beta$ treatment resulted in a significant reduction in the amount of type II collagen. However, overexpression of KLF2 significantly ameliorated the reduction of type II collagen (Fig. 7A). In contrast, silencing of KLF2 exacerbated the degradation of type II collagen (Fig. 7B).

\section{Discussion}

Previous studies have outlined that several signaling pathways and transcription factors have been implicated in inflammation associated with $\mathrm{OA}[17,18]$. The present in vitro study revealed that KLF2 prevented cartilage matrix degradation induced by the pro-inflammatory cytokine IL- $1 \beta$ in human primary chondrocytes. Firstly, we reported that KLF2 is expressed 


\section{Cellular Physiology Cell Physiol Biochem 2017;42:2159-2168 \begin{tabular}{l|l} 
and Biochemistry Published online: August 15, 2017 & $\begin{array}{l}\text { (c) } 2017 \text { The Author(s). Published by S. Karger AG, Basel } \\
\text { www.karger.com/cpb }\end{array}$
\end{tabular} \\ Yuan/Tan/Dai: KLF2 Regulates Degradation of Type II Collagen}

Fig. 7. KLF2 regulates the degradation of type II collagen. (A) Overexpression of KLF2 inhibited the degradation of type II collagen induced by IL- $1 \beta$ in human chondrocytes. The levels of collagen II in total cell lysates were determined by western blot analysis; (B) Knockdown of KLF2 promoted the degradation of type II collagen induced by IL-1 $\beta$ in human chondrocytes. The levels of collagen II in total cell lysates were determined by western blot analysis (ANOVA, ${ }^{*}, P<0.05$ vs. untreated cells (Ctr); \#, $P<0.05$ vs. IL$1 \beta$ group, $n=5-6)$.

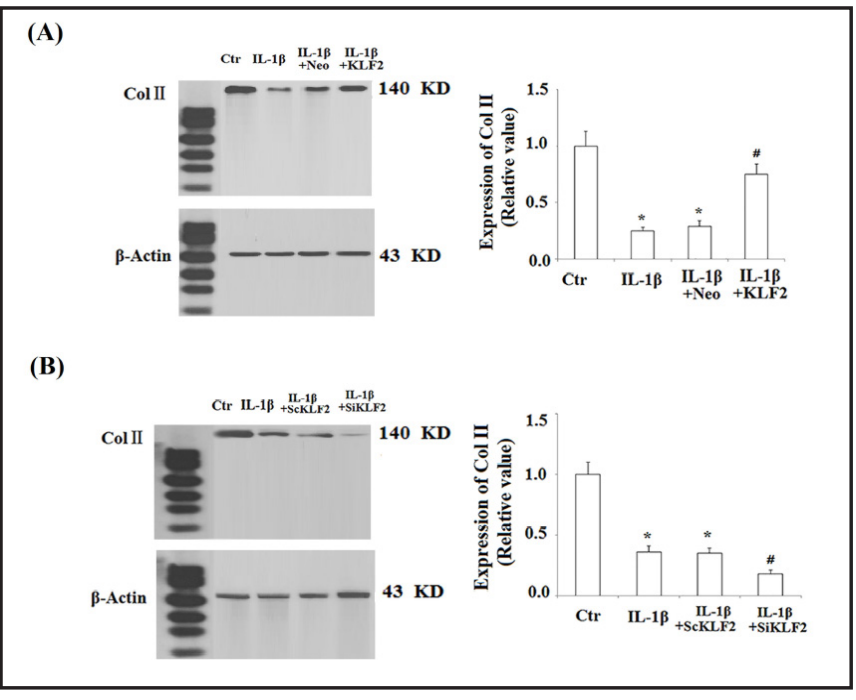

in primary chondrocytes and chondrogenic cell lines ATDC5 and OUMS27 cells for the first time. Secondly, we found that KLF2 was downregulated in chondrocytes from OA patients. Thirdly, KLF2 was found to suppress the expression of MMP-13 induced by IL-1 $\beta$. And lastly, KLF2 inhibited IL-1 $\beta$-induced type II collagen degradation.

Owing to its high expression in lung tissues, KLF2 was initially termed lung Krüppellike factor (LKLF). KLF2 is highly expressed in the vascular endothelium and has an antiinflammatory capacity [19]. Notably, KLF2 inhibits endothelial cell activation in response to diverse pro-inflammatory stimuli by affecting the common pro-inflammatory pathway nuclear factor (NF)-КB [20]. KLF2 has been found to suppress inflammation by downregulating MCP-1, VCAM, and TM and upregulating eNOS in endothelial cells [21]. Excessive expression of proinflammatory cytokines, such as tumor necrosis factor- $\alpha$ (TNF- $\alpha$ ) and IL-1 $\beta$, have been found in OA chondrocytes [22]. IL-1 $\beta$ has been widely used in in vitro models as an agent for OA induction [23]. The results of this study revealed for the first time that KLF2 is indeed expressed in primary chondrocytes. KLF2 may be downregulated in OA chondrocytes due to elevated levels of pro-inflammatory cytokines. Several previous studies have demonstrated that KLF2 could inhibit the production of inflammatory cytokines in different types of cells and tissues. It has been reported that myeloid KLF2 deficiency enhances the expression of IL-1 $\beta$ and TNF- $\alpha$ [24]. Importantly, KLF2 has been identified as a potent inhibitor of NF$\kappa \mathrm{B}$ activity and attenuates its ability to activate gene transcription [14]. NF- $\kappa \mathrm{B}$ is a central transcriptional factor that regulates inflammation-related signaling pathways in numerous diseases, including OA [25]. Studies have demonstrated that NF-кB triggers the expression of an array of genes that induce destruction of the articular joints, thereby leading to the onset and progression of OA. Interestingly, NF- $\kappa B$ has been reported to regulate the effects of IL- $1 \beta$ on the transcription of MMP-13 in human chondrocytes [26]. The anti-inflammatory capacity of KLF2 has been widely correlated to its inhibitory effects on the binding ability of NF- $\kappa \mathrm{B}$. Given the central role of NF- $\mathrm{BB}$ in modulating the expression of matrix metalloproteinases and pro-inflammatory cytokines, we speculated that KLF2 may regulate MMP-13 expression and degradation of collagen type II by modulating NF- $\kappa B$ activity. In addition, it is possible that KLF2 inhibits the expression of proinflammatory cytokines, such as IL- $1 \beta$ and TNF- $\alpha$, in human chondrocytes. Therefore, additional investigation is necessary to study the NF- $\mathrm{KB}$ / KLF2 axis and to elucidate the underlying mechanisms of KLF2's action on OA onset and progression.

Type II collagen is the major structural protein in cartilage and is also essentially unique to cartilaginous tissues. Our results demonstrate that KLF2 inhibits type II collagen by suppressing the expression of MMP-13, suggesting the potential role of KLF2 in the treatment of OA. In addition to MMPs, aggrecanases and other catabolic enzymes have been considered 


\section{Cellular Physiology Cell Physiol Biochem 2017;42:2159-2168 \begin{tabular}{ll|l} 
DOI: 10.1159/000479991 & $\begin{array}{l}\text { O 2017 The Author(s). Published by S. Karger AG, Basel } \\
\text { www.karger.com/cpb }\end{array}$ \\
\hline and Biochemistry Published online: August 15, 2017
\end{tabular}

to play an important role in the extracellular matrix, both directly and indirectly $[27,28]$. Investigations of the effects of KLF2 on the activity of these enzymes are ongoing in our laboratory. Pharmacological modifiers of KLF2 expression have been identified in previous studies. Further exploration of the underlying mechanisms may allow the introduction of these licensed pharmacological agents to be utilized for the treatment of OA. For example, statins have been widely used to treat primary and secondary coronary heart disease. Interestingly, multiple lines of evidence have shown that the atheroprotective effects and cholesterol-lowering abilities of statins are mediated by KLF2 [25]. Interestingly, mevastatin, a member of the statin family, has been proven to reduce inflammatory cell infiltration and cartilage degradation by suppressing the expression of IL-1 $\beta$, MMP-3, and MMP-13 in rabbit synovial tissues [26]. In addition, KLF2 has been reported to be involved in the antiinflammatory ability of montelukast, another licensed drug clinically used for the treatment of asthma [29]. However, it must be noted that there is a delicate balance between pro- and anti-inflammatory mechanisms in human joint cartilages [30]. KLF2 is the transcription factor that counter-balances the inflammatory response. Pharmacological intervention of KLF2 may easily disturb the inflammatory balance, resulting in the danger of unwanted sideeffects such as an impaired immune response.

To conclude, our findings suggest that KLF2 may be a potential therapeutic target for the treatment of OA. Further research will provide us with a more complete picture of the underlying mechanisms of KLF2 and other Krüppel family members in the pathological progression of $\mathrm{OA}$.

\section{Acknowledgements}

There is no funding for this work.

\section{Disclosure Statement}

The authors declare no competing financial or commercial interest that could be construed as a potential conflict of interest.

\section{Reference}

- Goldring MB: The role of the chondrocyte in osteoarthritis. Arthritis Rheum 2000;43:1916-1926.

2 Troeberg L, Nagase H: Proteases involved in cartilage matrix degradation in osteoarthritis. Biochim Biophys Acta 2012;1824:133-145.

-3 Mitchell PG, Magna HA, Reeves LM, Lopresti-Morrow LL, Yocum SA, Rosner PJ, Geoghegan KF, Hambor JE: Cloning, expression and type II collagenolytic activity of matrix metalloproteinase-13 from human osteoarthritic cartilage. J Clin Invest 1996;97:761-768.

-4 Chen S, Zhang L, Xu R, Ti Y, Zhao Y, Zhou L, Zhao J: TheBDKRB2 +9/-9 Polymorphisms Influence ProInflammatory Cytokine Levels in Knee Osteoarthritis by Altering TLR-2 Expression: Clinical and in Vitro Studies. Cell Physiol Biochem 2016;38:1245-1256.

-5 Xue H, Tu Y, Ma T, Liu X, Wen T, Cai M, Xia Z, Mei J: Lactoferrin Inhibits IL-1 $\beta$-Induced Chondrocyte Apoptosis Through AKT1-Induced CREB1 Activation. Cell Physiol Biochem 2015;36:2456-2465.

-6 Pelletier JP, Martel-Pelletier J, Abramson SB: Osteoarthritis, an inflammatory disease: potential implication for the selection of new therapeutic targets. Arthritis Rheum 2001;44:1237-1247.

7 Lim H, Kim HP: Matrix metalloproteinase-13 expression in IL-1 $\beta$-treated chondrocytes by activation of the p38 MAPK/c-Fos/AP-1 and JAK/STAT path-ways. Arch Pharm Res 201;34:109-117.

8 Li NG, Shi ZH, Tang YP, Wang ZJ, Song SL, Qian LH, Qian DW, Duan JA: New hope for the treatment of osteoarthritis through selective inhibition of MMP-13. Curr Med Chem 2011;18:977-1001. 


\section{Cellular Physiology Cell Physiol Biochem 2017;42:2159-2168 \begin{tabular}{l|l|l|l|}
\hline DOI: 10.1159/000479991 2017 The Author(s). Published by S. Karger AG, Basel & (c)
\end{tabular} and Biochemistry

-9 Chen WP, Xiong Y, Hu PF, Bao JP, Wu LD: Baicalein Inhibits MMPs Expression via a MAPK-Dependent Mechanism in Chondrocytes. Cell Physiol Biochem 2015;36:325-333.

10 Shields JM, Christy R.J, Yang VW: Identification and characterization of a gene encoding a gut-enriched Krüppel-like factor expressed during growth arrest. J Biol Chem 1996;271:20009-20017.

11 Dang DT, Pevsner J, Yang VW: The biology of the mammalian Krüppel-like family of transcription factors. Int J Biochem Cell Biol 2000;32:1103-1121.

-12 Kunes P, Lonsky’ V, Mand’ák J. Kolácková M, Kudlová M, Krejsek J: Transcription factor KLF2 (Krüppel-like factor 2) and natural defence of vascular endothelium. Cas Lek Cesk 2007;146:909-915.

-13 Suzuki T, Aizawa K, Matsumura T, Nagai R: Vascular implications of the Krüppel-like family of transcription factors. Arterioscler Thromb Vasc Biol 2005;25:1135-1141.

14 SenBanerjee S, Lin Z, Atkins GB, Greif DM, Rao RM, Kumar A, Feinberg MW, Chen Z, Simon DI, Luscinskas FW, Michel TM, Gimbrone MA Jr, Garcia-Cardena G, Jain MK: KLF2 is a novel transcriptional regulator of endothelial proinflammatory activation; J Exp Med 2004;199:1305-1315.

15 Hnasko TS, Hnasko RM: The Western Blot. Methods Mol Biol 2015;1318:87-96.

-16 Francin J, Abot A, Guillaume C, Moulin D,Bianchi A,Gegout-Pottie P,Jouzeau JY, Mainard D, Presle N: Association between adiponectin and cartilage degradation in human osteoarthritis. Osteoarthritis Cartilage 2014;22:519-526.

17 Wani MA, Conkright MD, Jeffries S, Hughes MJ, Lingrel JB: cDNA isolation, genomic structure, regulation, and chromosomal localization of human lung Krüppel-like factor. Genomics 1999;60:78-86.

18 Qi L, Tang Y, You Y, Qin F, Zhai L, Peng H, Nie R: Comparing the Effectiveness of Electroacupuncture with Different Grades of Knee Osteoarthritis: A Prospective Study. Cell Physiol Biochem 2016;39:2331-2340.

19 Feinberg MW, Lin Z, Fisch S, Jain MK: An emerging role for Krüppel-like factors in vascular biology. Trends Cardiovasc Med 2004;14:241-246.

-20 Kumar A, Lin Z, SenBanerjee S, Jain MK: Tumor necrosis factor alpha-mediated reduction of KLF2 is due to inhibition of MEF2 by NF-kappaB and histone deacetylases. Mol Cell Biol 2005;25:5893-5903.

-21 Atkins GB, Jain MK: Role of Krüppel-like transcription factors in endothelial biology. Circ Res 2007;100:1686-1695.

-22 Goldring MB, Otero M: Inflammation in osteoarthritis. Curr Opin Rheumatol 2011;23:471-478.

23 Liu Y, Wu D, Song F, Zhu C, Hui Y, Zhu Q, Wu J, Fan W, Hu J: Activation of $\alpha 7$ nicotinic acetylcholine receptors prevents monosodium iodoacetate-induced osteoarthritis in rats. Cell Physiol Biochem 2015;35: 27-638.

24 Mahabeleshwar GH, Kawanami D, Sharma N, Takami Y, Zhou G, Shi H, Nayak L, Jeyaraj D, Grealy R, White M, McManus R, Ryan T, Leahy P, Lin Z, Haldar SM, Atkins GB, Wong HR, Lingrel JB, Jain MK: The myeloid transcription factor KLF2 regulates the host response to polymicrobial infection and endotoxic shock. Immunity 2011;34:715-728.

-25 Sen-Banerjee S, Mir S, Lin Z, Hamik A, Atkins GB, Das H, Banerjee P, Kumar A, Jain MK; Kruppel-like factor 2 as a novel mediator of statin effects in endothelial cells. Circulation 2005;112:720-726.

-26 Akasaki Y, Matsuda S, Nakayama K, Fukagawa S, Miura H, Iwamoto Y: Mevastatin reduces cartilage degradation in rabbit experimental osteoarthritis through inhibition of synovial inflammation. Osteoarthritis Cartilage 2009;17:235-243.

-27 Pérez-García S, Gutiérrez-Cañas I, Seoane IV, Fernández J, Mellado M, Leceta J, Tío L, Villanueva-Romero R, Juarranz Y, Gomariz RP: Healthy and Osteoarthritic Synovial Fibroblasts Produce a Disintegrin and Metalloproteinase with Thrombospondin Motifs 4, 5, 7, and 12: Induction by IL-1 $\beta$ and Fibronectin and Contribution to Cartilage Damage. Am J Pathol 2016;186:2449-2461.

28 Du G, Song Y, Wei L, Li L, Wang X, Xu Q, Zhan H, Cao Y, Zheng Y, Ding D: Osthole Inhibits Proliferation and Induces Catabolism in Rat Chondrocytes and Cartilage Tissue. Cell Physiol Biochem 2015;36:2480-2493

29 Di X, Tang X, Di X: Montelukast inhibits oxidized low-density lipoproteins (ox-LDL) induced vascular endothelial attachment: An implication for the treatment of atherosclerosis. Biochem Biophys Res Commun 2017;486:58-62.

-30 Mobasheri A, Rayman MP, Gualillo O, Sellam J, van der Kraan P, Fearon U: The role of metabolism in the pathogenesis of osteoarthritis. Nat Rev Rheumatol 2017;13:302-311. 